\title{
Neck muscle atrophy and soft-tissue fibrosis after neck dissection and postoperative radiotherapy for oral cancer
}

\author{
Jinu Kim, PhD ${ }^{1,2}$, Eun Seow Shin ${ }^{3}$, Jeong Eon Kim³ , Sang Pil Yoon, MD, PhD'1, Young Suk Kim, MD ${ }^{4}$ \\ ${ }^{1}$ Department of Anatomy, Jeju National University School of Medicine, Jeju; \\ ${ }^{2}$ Department of Biomedicine \&t Drug Development, Jeju National University, Jeju; \\ ${ }^{3}$ Medical Course, Jeju National University School of Medicine, Jeju; \\ ${ }^{4}$ Department of Radiation Oncology, Jeju National University Hospital, Jeju National University School of Medicine, Jeju, Korea
}

Late complications of head and neck cancer survivors include neck muscle atrophy and soft-tissue fibrosis. We present an autopsy case of neck muscle atrophy and soft-tissue fibrosis (sternocleidomastoid, omohyoid, digastric, sternohyoid, sternothyroid, and platysma muscles) within the radiation field after modified radical neck dissection type I and postoperative radiotherapy for floor of mouth cancer. A 70-year-old man underwent primary tumor resection of the left floor of mouth, left marginal mandibulectomy, left modified radical neck dissection type $I$, and reconstruction with a radial forearm free flap. The patient received adjuvant radiotherapy. The dose to the primary tumor bed and involved neck nodes was $63 \mathrm{~Gy}$ in 35 fractions over 7 weeks. Areas of subclinical disease (left lower neck) received 50 Gy in 25 fractions over 5 weeks. Adjuvant chemotherapy was not administered.

Keywords: Autopsy, Neck muscles, Atrophy, Fibrosis, Radiotherapy, Adjuvant

\section{Introduction}

Radiation-induced toxicity is a major cause of long-term disability after head and neck cancer treatment, such as subcutaneous soft-tissue fibrosis, neck muscle atrophy, swallowing abnormality, and trismus [1-4]. Radiotherapy is often combined with surgery and/or chemotherapy; therefore, the toxicities of these modalities may be cumulative and difficult to separate clinically [1]. Neck muscle atrophy and soft-tissue fibrosis have received little attention in the clinical literature. We report a morphological analysis of neck muscle atrophy and soft-tissue fibrosis in an oral cancer autopsy case after modified radical neck dissection (MRND) type I and adjuvant radiotherapy to gain more insight into the clinical picture of neck muscle atrophy and soft-tissue fibrosis.

\section{Case Report}

A 70-year-old man presented with a 5-month history of an ulcerative left floor of mouth mass and a left neck mass. His history was unremarkable. Physical examination revealed an ulcerative and exophytic mass in the left floor of mouth without extension across the midline. A 5-cm node was palpated in the left subdigastric region. He was referred to a tertiary institution for workup and treatment. A biopsy from the floor of mouth lesion showed squamous cell carcinoma with moderate differentiation. The patient underwent primary tumor resection of the left floor of mouth via pull through

Received 24 September 2015, Revised 3 December 2015, Accepted 8 December 2015.

Correspondence: Young Suk Kim, MD, Department of Radiation Oncology, Jeju National University Hospital, Jeju National University School of Medicine, 15 Aran 13-gil, Jeju 63241, Korea. Tel: +82-64-717-1330, Fax: +82-64-717-1169, E-mail: yskim@ jejunuh.co.kr

(c) This is an Open Access article distributed under the terms of the Creative Commons Attribution Non-Commercial License (http://creativecommons.org/ licenses/by-nc/4.0/) which permits unrestricted non-commercial use, distribution, and reproduction in any medium, provided the original work is properly cited.

www.e-roj.org 
approach, left marginal mandibulectomy, left MRND type I, and reconstruction with a radial forearm free flap. MRND type I included removal of nodal levels I through $V$, the left internal jugular vein (IJV), and part of the sternocleidomastoid (SCM) muscle (clavicular head) due to muscle invasion, sparing the spinal accessory nerve (SAN). The product of MRND measured $11.5 \mathrm{~cm} \times 7 \mathrm{~cm} \times 3.5 \mathrm{~cm}$. Histopathological analysis revealed a $2.5 \mathrm{~cm} \times 1.5 \mathrm{~cm} \times 1.3 \mathrm{~cm}$ squamous cell carcinoma with two lymph node metastases of 58 cervical lymph nodes. Extracapsular extension was observed in 2-cm-sized metastatic lymph node. The deep resection margin was close (less than $0.1 \mathrm{~cm}$ ). The pathological stage was T2N2bM0 according to the American Joint Committee on Cancer staging system, 7th edition.

The patient received adjuvant radiotherapy 5 weeks after the operation. The primary tumor bed and left upper neck nodes were treated using $4 \mathrm{MV}$ photons. The dose to the primary tumor bed and involved neck nodes was 63 Gy in 35 fractions over 7 weeks with two cone-down treatment plans at $45 \mathrm{~Gy}$ and $61.2 \mathrm{~Gy}$. Areas of subclinical disease (left lower neck) received 50 Gy in 25 fractions over 5 weeks. Adjuvant chemotherapy was not administered.

He complained of dry mouth 1 year and 6 months after radiotherapy. Physical examination revealed hardness of the left neck, which continued to exist without remarkable improvement. He complained about dysphagia at 3 years and 7 months after radiotherapy. His dysphagia was stationary at 3 years and 11 months after radiotherapy. He complained about a decrease in tongue movement at 4 years and 4 months after radiotherapy. Physical examination revealed an adhesion on the left floor of mouth tumor bed. Subclinical hypothyroidism was diagnosed 5 years after radiotherapy.

He remained without evidence of disease 7 years and 6 months after radiotherapy. A mass was found in the right lower lung field on chest $X$-ray examination at that time. Chest computed tomography (CT) showed multiple pulmonary metastases. The patient's family refused further treatment because of old age. He died of progressive pulmonary metastasis and right pleural effusion 9 years and 5 months after radiotherapy. The primary tumor bed and neck node areas
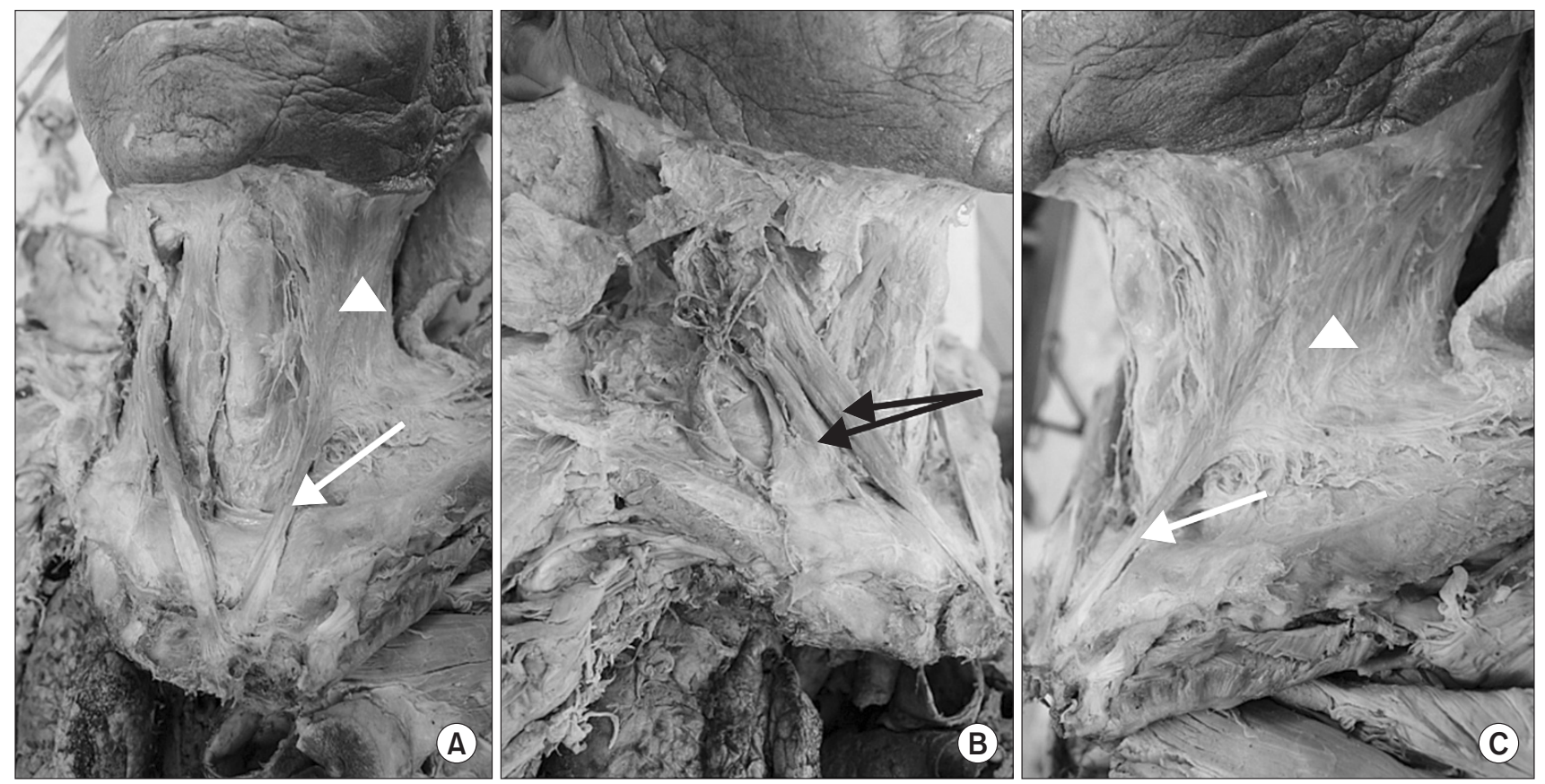

Fig. 1. Atrophy and soft-tissue fibrosis in the left sternocleidomastoid (SCM), omohyoid, digastric (two bellies), sternohyoid, sternothyroid, and platysma muscles in left nodal levels I through IV on autopsy. (A) Anterior, (B) right lateral, and (C) left lateral view of the superficial neck muscle layer. $(A, C)$ The white arrow indicates the left sternal head of the SCM muscle. (B) The black arrow indicates the right sternal and clavicular heads of the SCM muscle. The clavicular head of left SCM muscle was removed during neck dissection due to muscle invasion. The left platysma muscle is seen as a thin fibrosis membrane due to severe atrophy and fibrosis. ( $A, C)$ Muscles (the left SCM, omohyoid, digastric [two bellies], sternohyoid, sternothyroid, and platysma) and fascia joined together as a unit (white arrow head) (Common Terminology Criteria for Adverse Events ver. 3.0 grade 3 fibrosis of deep connective tissue). Left deep neck muscles were intact in comparison with right muscles (data not shown). 
showed no evidence of disease. His family donated his body to our medical school.

We began to dissect the cadaver to teach human anatomy 2 years and 9 months after his death. On dissection, muscle atrophy and soft-tissue fibrosis were severe in the left SCM, omohyoid, digastric (two bellies), sternohyoid, sternothyroid, and platysma muscles (Fig. 1). Common Toxicity Criteria ver. 3.0 grade 3 fibrosis of the subcutaneous tissue, which is characterized by a marked increase in tissue density with retraction or fixation of the subcutaneous tissue layer on the deep tissue layer, was observed in left neck nodal levels I through IV [5]. Grade 3 fibrosis of deep connective tissue (with increased density and thickness that is distinctive from grade 2 fibrosis characterized by fixation of the tissue layers causes

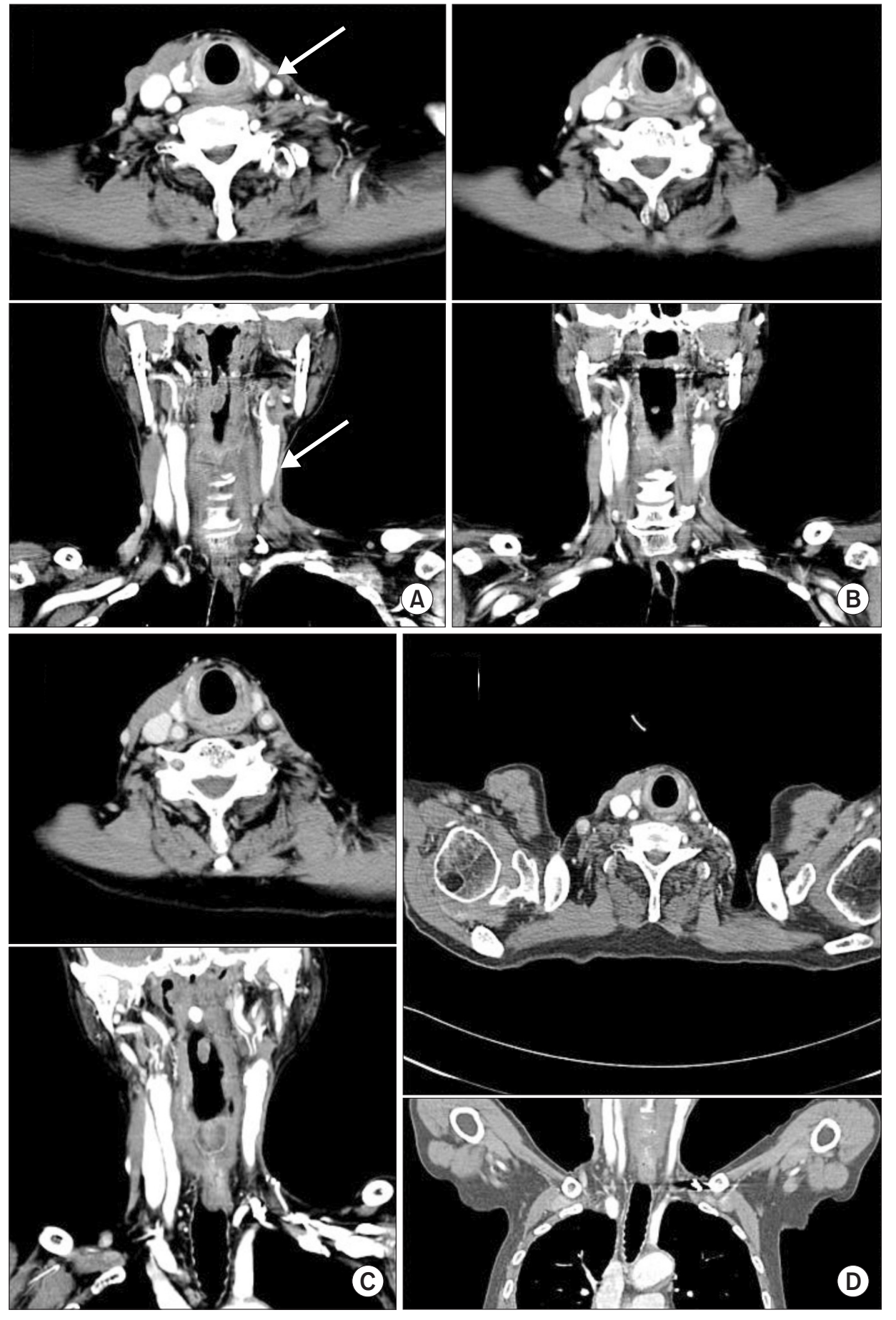

Fig. 2. Serial computed tomography (CT) images of the patient on coronal and axial imaging. Atrophy of the left sternocleidomastoid muscle is prominent in the left irradiated field (white arrow). The patient has a marked decrease in fat beneath the left sternocleidomastoid muscle. This resulted in the muscle directly abutting the paraspinal muscles. The left internal jugular vein was removed during neck dissection. (A) Two years and 11 months, (B) 5 years and 1 month, (C) 7 years, and (D) 9 years and 2 months after radiotherapy. The $\mathrm{CT}$ image of (D) is a little different from the others due to the chest CT image. The chest CT was performed to evaluate pulmonary metastasis.

http://dx.doi.org/10.3857/roj.2015.33.4.344 
them to move as a single unit) was observed in the left neck nodal levels I through IV [5]. Muscles (the left SCM, omohyoid, digastric [two bellies], sternohyoid, sternothyroid, and platysma) and fascia moved as a unit. We also retrospectively reviewed the $\mathrm{CT}$ images of the patient. Contrast-enhanced CT of the neck showed atrophy of the whole left SCM muscle. Representative images are presented at the cricoid cartilage level in Fig. 2.

\section{Discussion}

In this case, we found on autopsy, neck muscle atrophy and soft-tissue fibrosis in the radiation field after left MRND type I and adjuvant radiotherapy on autopsy. Postoperative radiotherapy was combined with neck dissection; therefore, the toxicities of these modalities may be cumulative and difficult to separate clinically [1].

The MRND is a modification of the radical neck dissection that preserves one or more of the functional or nonlymphatic structures, including the SCM muscle, SAN, or IJV [6]. Specifically, type I MRND preserves the SAN, type II MRND preserves the SAN and IJV, and type III MRND preserves the SAN, IJV, and SCM. In our case, the patient underwent left MRND type I, including removal of nodal levels I through

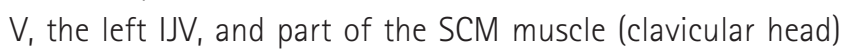
due to muscle invasion, sparing the SAN. Resection of the primary tumor and neck dissection prior to radiotherapy can significantly and adversely affect local structures and increase toxicity $[1,4]$. Hirota et al. [4] reported that previous neck dissection is a significant prognostic variable for moderate to severe subcutaneous fibrosis after neck irradiation. Wratten et al. [7] assessed the effect of surgery on normal tissue toxicity in head and neck cancer patients treated with accelerated radiotherapy. They found significantly more subcutaneous fibrosis in the surgery plus postoperative radiotherapy group ( $p<0.01$ ) than the definitive radiotherapy group (grade 3 , or more, late toxicity at 5 year; $34 \%$ vs. 16\%) [7]. Analysis of late toxicity showed an increase in subcutaneous fibrosis in the postoperative group even though the total dose was less in this group (mean dose: 52.96 Gy vs. 59.27 Gy) [7].

Primary tumor resection and neck dissection alone without adjuvant radiotherapy can also induce neck muscle atrophy and shoulder weakness [8-10]. Cuccia et al. [10] reported significant SCM muscle atrophy on the side of MRND type III $(p<0.05)$ with no radiotherapy in 45 head and neck cancer patients. The atrophy was particularly marked in the caudal and middle portions of the SCM muscle and was likely related to damage to the segmental blood supply in the caudal and middle portions of the muscle as well as injury to innervations [10]. The blood supply to the SCM muscle has been well described [11]. Comparing the patterns of muscle atrophy, which were significantly marked at the middle and lower sections, Cuccia et al. [10] suggested that the most frequent sites of damages are at the superior thyroid artery and the subclavian branch.

One of the most common complications of neck dissection is injury to the SAN [8]. Injury to the SAN can cause atrophy of the SCM and trapezius muscles [8]. Significant incidence of SAN injury has been reported that might have resulted from nerve manipulation has been reported, although the SAN was preserved during neck dissection $[8-10,12]$. Cappiello et al. [9] reported patients treated with SAN-sparing neck dissection (selective neck dissection) may present evident signs of SCM and trapezius muscle dysfunction. Cuccia et al. [10] described possible intraoperative damage to the SCM muscle innervations during MRND type III in head and neck cancer patients: 1) damage to the SAN trunk; 2) damage to the SAN branch; 3) local damage to the fibers running through the SCM muscle; and 4) damage to the SCM branch of the cervical nerve.

Peripheral nervous system dysfunction can also result from external compressive fibrosis of soft tissues, ischemia caused by fibrosis and subsequent compromise of the vasa vasorum, or both [1]. Pain, sensory loss, and weakness are the most commonly observed clinical features of peripheral nervous system dysfunction [1].

Radiation-induced myopathy has been reported as a product of radiotherapy of head and neck cancers [13-15]. Delayed lesions are found six months after irradiation and worsen progressively for years thereafter. Atrophy of the remaining viable muscle cells is prominent, as is mild or severe interstitial fibrosis $[5,16]$. On occasion, there is massive fibrosis of an entire muscle group [16]. In our case, muscles (the left SCM, omohyoid, digastrics [two bellies], sternohyoid, sternothyroid, and platysma) and fascia moved as a unit (Fig. 1). The late effect may have resulted from a combination of vascular damage and the loss of skeletal muscle parenchymal cells [17]. Damage of the endothelium and smooth muscle of the intramuscular blood vessels may have contributed to muscle ischemia $[14,18]$. A number of microscopic features of radiationinduced injury to skeletal muscle include: 1) close-packed groups of round, hyperchromatic nuclei lying in the center of the granular eosinophilic muscle cytoplasm, which resemble epithelium more than muscle cells; 2) some of the cells 
contain two, three, or more nuclei; 3) in some areas, bundles of nearly normal striated muscle fibers merge into groups of bizarre multinucleated giant cells, which are often considered regenerating but also possibly degenerating skeletal muscle cells; 4) foci of inflammatory cell infiltrates are seen; 5) broad areas of dense fibrosis are also prominent; 6) fibroblasts with a large, hyperchromatic nucleus are seen in the areas of fibrosis; 7) small blood vessels, probably arterioles or small arteries, with greatly thickened walls are found; and 8) a few dilated, thinwalled vessels suggesting telangiectasia are noted [16]. In our case, it was difficult to find gross blood vessel changes in the radiation field (Fig. 1). The common presentations of radiationinduced myopathy include neck extensor weakness, shoulder pain and dysfunction, and trismus $[1,14]$.

Many studies reported neck muscle atrophy and soft-tissue fibrosis after neck dissection and postoperative radiotherapy in head and neck cancer patients $[4,14]$. Two-dimensional or three-dimensional conformal radiotherapy was mainly used in these patients. Recently, intensity-modulated radiotherapy (IMRT) has been commonly used in head and neck cancer patients [19]. Also, involved-field radiotherapy is performed in general rather than extended fields of irradiation to reduce the risk of late complications in lymphoma patients [13]. IMRT and involved-field radiotherapy could reduce neck muscle atrophy and soft-tissue fibrosis by minimizing radiation dose to normal tissues.

Previous studies have reported a considerable time lag between the exposure to radiation and development of the myopathy with a range of several months to years (range, 2 to 45 years) $[13,14]$. In our case, serial CT follow-up images showed atrophy of the SCM muscle (Fig. 2). At the last followup (Fig. 2D), the patient was 79 years old with multiple pulmonary metastases, and he died of respiratory failure 2 months later. Old age and a metastatic condition can cause some confusion regarding whether the case represents the progression of neck muscle atrophy or a natural course of aging and cachexia from pulmonary metastasis.

van Leeuwen-Segarceanu et al. [20] reported progressive muscle atrophy and weakness after treatment with mantle field radiotherapy in 12 Hodgkin Iymphoma survivors. Based on their hypothesis that primary vascular injuries may cause myogenic damage in the muscles in the radiation field, they recommend training the affected muscles. It is hoped that this may promote increased vascularization of the muscles through the formation of collateral vessels, which can contribute to maintaining muscle strength and integrity.

Our study has several limitations. Isodose coverage of the postoperative tumor bed and neck area was not described because of the lack of details concerning treatment planning data. Preoperative CT images were not available. There was limited long-term follow-up data concerning late complications due to the retrospective nature and low patient compliance. We did not report pathological findings of irradiated neck muscles. Pathological studies may have strengthened our report.

To our knowledge, our report is the first to describe neck muscle atrophy and soft-tissue fibrosis after primary neck dissection and postoperative radiotherapy in an autopsy case. Clinicians need to be aware that neck muscle atrophy and softtissue fibrosis can occur in head and neck cancer survivors who have previously undergone MRND and postoperative neck irradiation.

\section{Conflict of Interest}

No potential conflict of interest relevant to this article was reported.

\section{Acknowledgments}

The authors are grateful to Department of Radiation Oncology, Korea University College of Medicine, Seoul, Korea. This institute provided patient treatment information.

\section{References}

1. Stubblefield MD. Radiation fibrosis syndrome: neuromuscular and musculoskeletal complications in cancer survivors. Pm R 2011;3:1041-54.

2. Portlock CS, Boland P, Hays AP, Antonescu CR, Rosenblum MK. Nemaline myopathy: a possible late complication of Hodgkin's disease therapy. Hum Pathol 2003;34:816-8.

3. Citrin D, Mansueti J, Likhacheva A, et al. Long-term outcomes and toxicity of concurrent paclitaxel and radiotherapy for locally advanced head-and-neck cancer. Int J Radiat Oncol Biol Phys 2009;74:1040-6.

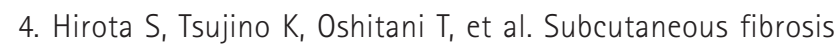
after whole neck irradiation. Int J Radiat Oncol Biol Phys 2002; 52:937-43.

5. Davis AM, Dische S, Gerber L, Saunders M, Leung SF, O'Sullivan B. Measuring postirradiation subcutaneous soft-tissue fibrosis: state-of-the-art and future directions. Semin Radiat Oncol 2003;13:203-13.

6. Hudgins PA, Kingdom T, Weissler MC, Mukherji SK. Selective neck dissection: $C T$ and MR imaging findings. AJNR Am J 
Neuroradiol 2005;26:1174-7.

7. Wratten CR, Poulsen MG, Williamson S, Tripcony L, Keller J, Dickie $G$. Effect of surgery on normal tissue toxicity in patients treated with accelerated radiotherapy. Acta Oncol 2002;41:5662.

8. Cheng PT, Hao SP, Lin YH, Yeh AR. Objective comparison of shoulder dysfunction after three neck dissection techniques. Ann Otol Rhinol Laryngol 2000;109(8 Pt 1):761-6.

9. Cappiello J, Piazza C, Giudice M, De Maria G, Nicolai P. Shoulder disability after different selective neck dissections (levels II-IV versus levels II-V): a comparative study. Laryngoscope 2005; 115:259-63.

10. Cuccia G, Shelley OP, d'Alcontres FS, Giannitrapani M, Soutar DS, Camilleri IG. Evidence of significant sternocleidomastoid atrophy following modified radical neck dissection type III. Plast Reconstr Surg 2006;117:227-32.

11. Kierner AC, Aigner M, Zelenka I, Riedl G, Burian M. The blood supply of the sternocleidomastoid muscle and its clinical implications. Arch Surg 1999;134:144-7.

12. Kelley MJ, Kane TE, Leggin BG. Spinal accessory nerve palsy: associated signs and symptoms. J Orthop Sports Phys Ther 2008;38:78-86.

13. Furby A, Behin A, Lefaucheur JP, et al. Late-onset cervicoscapular muscle atrophy and weakness after radiotherapy for Hodgkin disease: a case series. J Neurol Neurosurg Psychiatry 2010;81:
101-4.

14. Ghosh PS, Milone M. Clinical and laboratory findings of 21 patients with radiation-induced myopathy. J Neurol Neurosurg Psychiatry 2015;86:152-8.

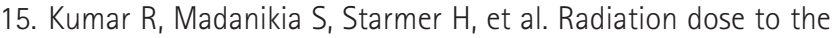
floor of mouth muscles predicts swallowing complications following chemoradiation in oropharyngeal squamous cell carcinoma. Oral Oncol 2014;50:65-70.

16. Fajardo LF, Berthrong $M$, Anderson RE. Radiation Pathology. New York, NY: Oxford University Press; 2001.

17. Hall EJ, Giaccia AJ. Radiobiology for the radiologist. 6th ed. Philadelphia, PA: Lippincott Williams \& Wilkins; 2006.

18. Veinot JP, Edwards WD. Pathology of radiation-induced heart disease: a surgical and autopsy study of 27 cases. Hum Pathol 1996;27:766-73.

19. Van Gestel D, Van Den Weyngaert D, Schrijvers D, Weyler J, Vermorken JB. Intensity-modulated radiotherapy in patients with head and neck cancer: a European single-centre experience. Br J Radiol 2011;84:367-74.

20. van Leeuwen-Segarceanu EM, Dorresteijn LD, Pillen S, Biesma DH, Vogels OJ, van Alfen N. Progressive muscle atrophy and weakness after treatment by mantle field radiotherapy in Hodgkin Iymphoma survivors. Int J Radiat Oncol Biol Phys 2012;82:612-8 